\title{
Modelling human behaviour in a bumper car ride using molecular dynamics tools: a student project
}

\author{
Jorge J Buendía ${ }^{1}$, Hector Lopez ${ }^{1}$, Guillem Sanchis ${ }^{1}$ and \\ Luis Carlos Pardo ${ }^{2}$ \\ ${ }^{1}$ Universitat Politècnica de Catalunya, Escola Tècnica Superior d'Enginyeria de \\ Telecomunicacions de Barcelona, Barcelona, Catalonia, Spain \\ ${ }^{2}$ Grup de Caracterització de Materials, Departament de Física, EEBE, Universitat \\ Politècnica de Catalunya, C/ Eduard Maristany 10-14, E-08930, Barcelona, Catalonia, \\ Spain \\ E-mail: luis.carlos.pardo@upc.edu
}

Received 20 December 2016, revised 20 February 2017

Accepted for publication 7 March 2017

Published 30 March 2017

\begin{abstract}
Amusement parks are excellent laboratories of physics, not only to check physical laws, but also to investigate if those physical laws might also be applied to human behaviour. A group of Physics Engineering students from Universitat Politècnica de Catalunya has investigated if human behaviour, when driving bumper cars, can be modelled using tools borrowed from the analysis of molecular dynamics simulations, such as the radial and angular distribution functions. After acquiring several clips and obtaining the coordinates of the cars, those magnitudes are computed and analysed. Additionally, an analogous hard disks system is simulated to compare its distribution functions to those obtained from the cars' coordinates. Despite the clear difference between bumper cars and a hard disk-like particle system, the obtained distribution functions are very similar. This suggests that there is no important effect of the individuals in the collective behaviour of the system in terms of structure. The research, performed by the students, has been undertaken in the frame of a motivational project designed to approach the scientific method for university students named FISIDABO. This project offers both the logistical and technical support to undertake the experiments designed by students at the amusement park of Barcelona TIBIDABO and accompanies them all along the scientific process.
\end{abstract}

Supplementary material for this article is available online 
Keywords: human behaviour, bumper cars, liquids, radial distribution function, colisions, molecular dynamics simulation

(Some figures may appear in colour only in the online journal)

\section{Introduction}

The laws of physics come to life in an amusement park where physical magnitudes can be measured and their relation can be checked in extreme conditions. However, the measurement of such magnitudes is difficult because this environment cannot be controlled either by students or teachers. The opposite happens in a 'classical' laboratory at schools and universities: the experiments are performed in a completely controlled way. This leads to the problem that difficulties, both in the design of an experiment and in the data analysis, remain hidden in those rather artificial situations. Students thus wrongly conclude that experiments usually work at a first instance, and that the analysis of data is straightforward. Moreover, the most important part of research, creativity, too often plays no role in standard university curricula. To overcome these two problems, we offer first year students studying the Physics Engineering degree from the 'Escola Tècnica Superior d'Enginyeria de telecomunicacions de Barcelona' at 'Universitat Politècnica de Catalunya' (UPC) the chance to participate in a motivational activity called FISIDABO ${ }^{3}$. Below we present a summarized description of this project.

First of all we propose to the students the idea of sending a project to a scientific board, composed by scientists teaching the Physics Engineering degree at UPC. It is important to emphasize that some members of the scientific board perform experiments at large facilities such as synchrotrons or neutron sources on a regular basis. The projects are presented by teams of a maximum three persons. The goal and description of the experiments must be clearly explained by the students in their proposals. The projects are then selected taking into account their scientific quality, originality and feasibility. Once the projects are chosen, a tutor is assigned to each team, to guide the students on technical issues and to assure the feasibility of the experiment. To start developing the experiments, in a day when the amusement park is open to the public, the students perform preliminary experiments that do not interfere with the normal activity of the park. On a second day, the amusement park is opened exclusively for the students participating in the project. During this day the students perform the experiments using the rides at the TIBIDABO amusement park.

The teams must afterward write a short experimental report summarizing the preliminary results of their experiment. In a final stage, these results must be carefully analyzed and, depending on the quality of their research, they are encouraged to write a paper to the same standards as those of any scientific paper (such as the one presented in this journal). These final steps (analysis and paper writing) are part of what might be called the 'hidden' work of a scientist, and are crucial in scientific research: too often students have the wrong impression that experiments always lead directly to the answer sought by the experiment, and they ignore the importance of sharing the results with the scientific community. We would like to point out that this procedure is analogous to the process that takes place when scientists aim at using a large scientific facility such as a neutron source or a synchrotron. This is not by chance: the goal of the project is to approach the 'real' scientific method as much as possible

3 Videos and a description of the FISIDABO project can be found at www.fisidabo.org. The video related to this specific experiment can be found at www.youtube.com/watch?v=3f91YLhpSCE 


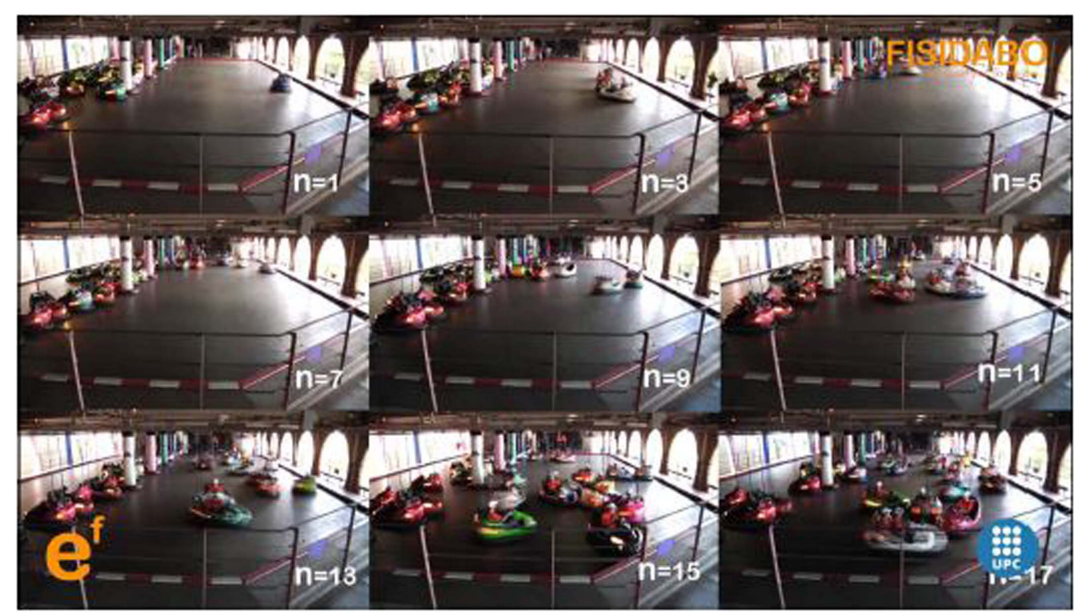

(a)
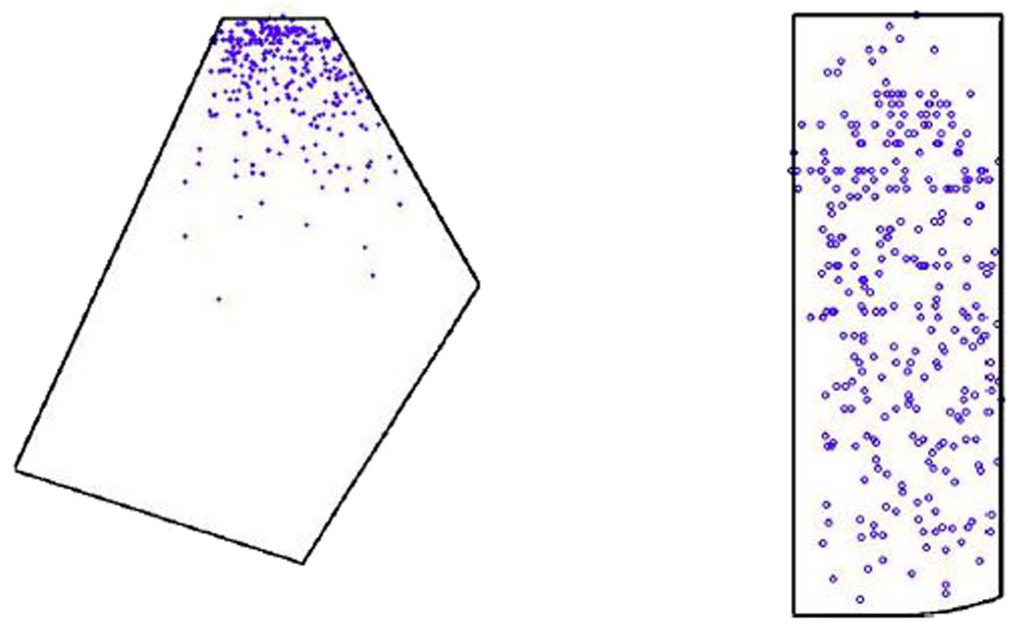

(b)

Figure 1. (a) Some frames of the footage used, with different numbers of cars. Notice the important distortion due to the perspective. (b) Example of the cars' coordinates as directly obtained from the footage (left) and once the perspective distortion has been corrected (right).

to students, so that they find the same problems and satisfactions as they would in a professional research career.

The project presented in this work was selected by the scientific board, with the first three authors the students that performed the experiment. The last author is the tutor, currently working in the field of chemical physics, and a frequent user of several large facilities such as the neutron sources ILL in Grenoble (France), ISIS in Didcot (UK) and MLZ in Garching (Germany). The goal of the project presented in this paper is, though, not directly related to physics, but to find out if statistical tools that are used in analyzing molecular trajectories (as those obtained from a simulation) can shed some light on human behaviour when driving 
bumper cars. This project has the advantage that it can be performed at any place where a bumper car ride is to be found.

Emergent phenomena arising from the collective motion of humans and, in general, of many different self-propelled and often living entities, have been studied in many specific social settings [1]. Examples of these phenomena are the spontaneous arising of certain behaviours, such as unidirectional lanes or stop-and-go lanes in pedestrian traffic [2], the socalled Mexican waves among spectators at sporting events [3], vortex- and gas-like structures during heavy metal concerts [4], or behaviour during escape from a disaster [5-7]. Some of the aforementioned works $[1,2,4]$ reach the interesting conclusion that often human behaviour can be predicted from surprisingly simple rules and models. However, all these previous works focus on the dynamical properties of the respective systems and, as far as we know, little is done in obtaining structural features on such human-driven systems. Instead, in this work we focus on obtaining information, to our knowledge for the first time, about the structural features arising from collective human behaviour, and compare our results with a system as simple as that of hard disks [8, 9].

The aim of this paper is to study the structural properties of a system composed of bumper cars driven by human beings. In particular, the interest is in finding what the effect is, if any, of the fact that the drivers are conscious beings, in contrast with simple particle systems.

\section{Methods}

\subsection{Obtaining particle trajectories}

The experiment was carried out in the TIBIDABO amusement park in Barcelona. No previous information about the goal of the experiment was given to the participants to avoid bias in the results. The number of drivers was increased from ride to ride in order to investigate the system with a significant range of densities, from as little as three cars (meaning most of the track is empty) to 25 (at which point cars tend to get clustered together simply because they have little space available to move). To study the motion of the bumper cars, a static camera was placed in a centred and elevated position with respect to the track. In this way, several videos were recorded, with the variable being the number of cars participating (figure 1). The dimensions of the track are $10 \mathrm{~m} \times 29 \mathrm{~m}$. The dimensions of the cars are $115 \mathrm{~cm} \times 192 \mathrm{~cm}$. The obtained footage consisted of several video clips of about $140 \mathrm{~s}$ duration. Each clip contains a constant number of cars. Data corresponding to static coordinates (i.e. the coordinates of the cars at each frame, but no information about their trajectories) were then obtained from the footage using the Manual Tracking plug-in for ImageJ ${ }^{4}$. Specifically, the coordinates of the cars were extracted from snapshots every $2 \mathrm{~s}$, meaning around 70 datasets per clip.

Due to the perspective of the camera, the first step prior to any data analysis was to correct this effect in the obtained coordinates. In order to do that we measured the coordinates of some spots in the crash cars track $\left(x_{t}, y_{t}\right)$, and we compared them with the obtained coordinates of such spots recorded with the camera $\left(x_{c}, y_{c}\right)$. The transformation for the $x$ coordinate was found to be linear, but that was not the case for the $y$-coordinate. Although the transformation in the $y$-axis could have been calculated from trigonometric considerations, we used a heuristic approach, and we found that an exponential transformation yielded an excellent result for the correction of the perspective effect in the $y$-axis (see figure 1). The

${ }^{4}$ http://rsbweb.nih.gov/ij/plugins/track/track.html 

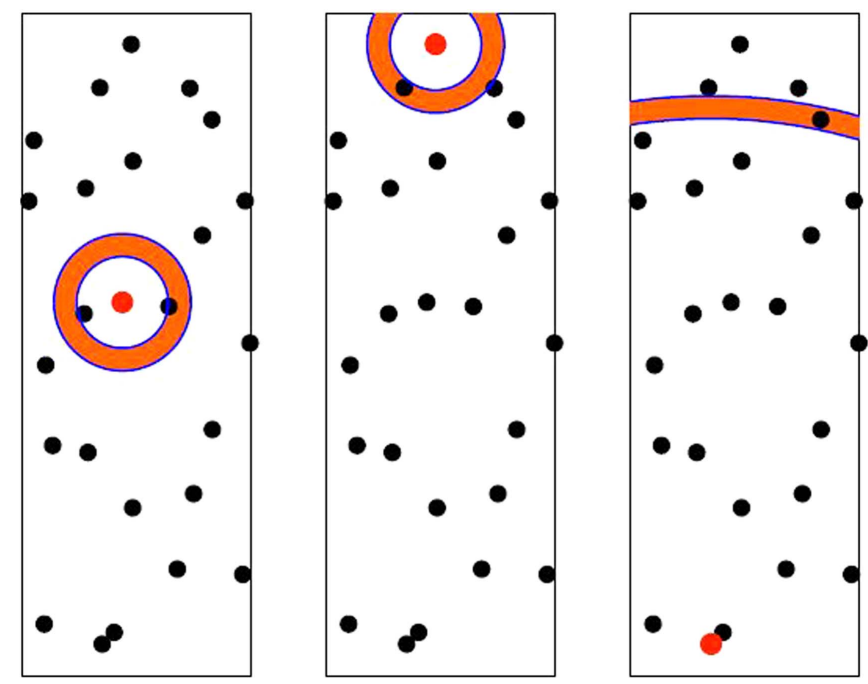

Figure 2. Points show the position of bumper cars and shaded areas show the regions used to calculate the RDF. Left panel show the ideal situation in which the region used to calculate the RDF is fully contained in the track. Central and right panels show the cases in which a correction must be performed to account for the limited region used to count bumper cars at a certain distance range (see text for details).

obtained transformation function is

$$
\left[x_{t}, y_{t}\right]=\left[x_{c}-y_{c} \cdot \frac{x_{\max }-x_{c}}{y_{\max }-y_{c}}, e^{\left(0.01 \cdot y_{c}\right)}\right]
$$

with $x_{c}$ and $y_{c}$ the coordinates obtained by the camera, $x_{t}$ and $y_{t}$ the real coordinates of the cars in the track, and $x_{\max }$ and $y_{\max }$ the coordinates of the contours of the track.

\subsection{Simulation of a hard-disks fluid}

The simulations correspond to hard disks-like particle systems. In this kind of simulation, the particles are modelled as rigid circles (as an analogy of the case of rigid spheres in threedimensional simulations) characterized by a mass and a radius. These particles only interact via elastic collisions, so that the kinetic energy is conserved along the simulation. Formally, this interaction corresponds to a potential of the form

$$
V(r)=\left\{\begin{array}{ll}
0 & r>r_{0} \\
\infty & r \leqslant r_{0}
\end{array} .\right.
$$

Here, $r_{0}$ is an effective radius which we define as the radius of a circle with the same surface area as the car (as projected on the ground). In this simulation, it is taken to be $75 \mathrm{~cm}$.

As stated before, no periodic boundary conditions are imposed. The particles in the simulation are confined in a box with the same dimensions as the track $(1000 \mathrm{~cm} \times 2900$ $\mathrm{cm})$. Collisions with the walls are assumed to be elastic as well.

The maximum time step for this simulation is taken as $\Delta t=0.05 \mathrm{~s}$. This value is the time step after which the coordinates of the particles are updated if the particle does not collide with another one. In the event of a collision, we calculate the time when it happens, and update the coordinates and velocities accordingly. Collisions in this time lapse are easily 

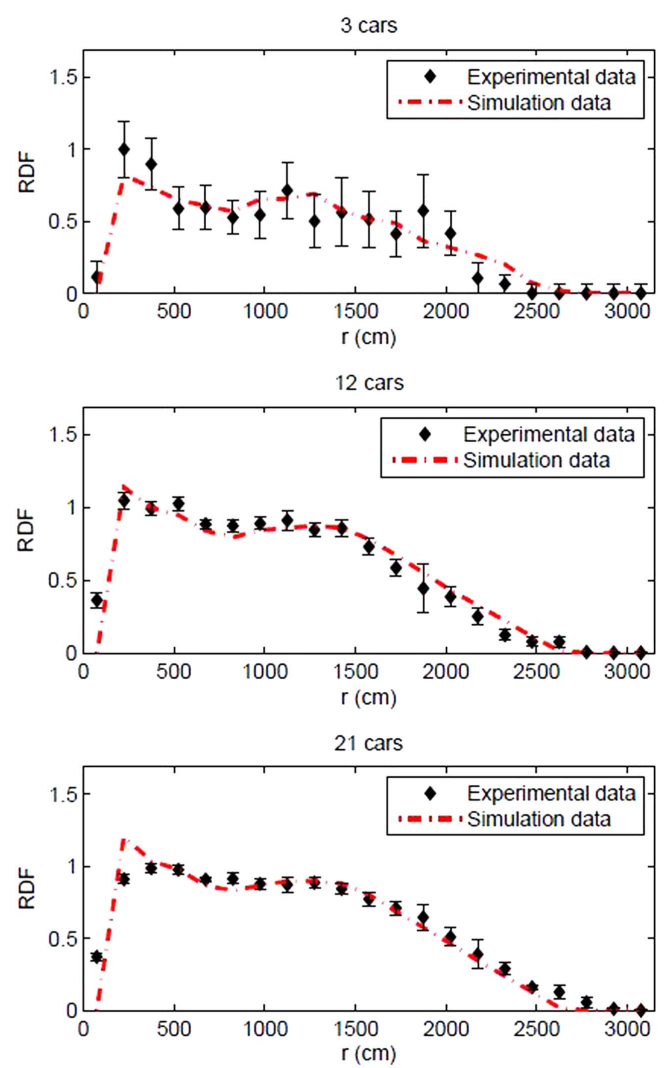

Figure 3. RDF for 3 (top), 12 (middle) and 21 (bottom) cars. Dash-dotted lines correspond to the simulations.

predicted since in the absence of external forces, the particles follow straight trajectories with constant speed. Test runs with smaller time increments have been done without noticeable changes in the distribution functions.

\section{Data analysis}

Data analysis includes the computation of the radial and angular distribution functions, two correlation functions frequently used in molecular dynamics.

The radial distribution function (RDF) was calculated in a standard way, computing the distances between all particle pairs in each frame and binning them into a histogram, with the corresponding normalization. This normalization accounts for the fact that, even with no correlation between particles (as in an ideal gas), the likelihood of finding particles at larger distances is greater, simply because we are taking into account more volume (or surface area, in our case).

Regarding this normalization, it is worth noting that, since in this case the system is finite in size (i.e. no periodic boundary conditions are applied), the normalization factor has to take into account only the actual area within the boundaries. Of course, this is only important when particles are close to the edges (see figure 2, central panel) and/or at larger distances, i.e. those comparable to the size of the box (see figure 2, right panel). In these two cases only the 

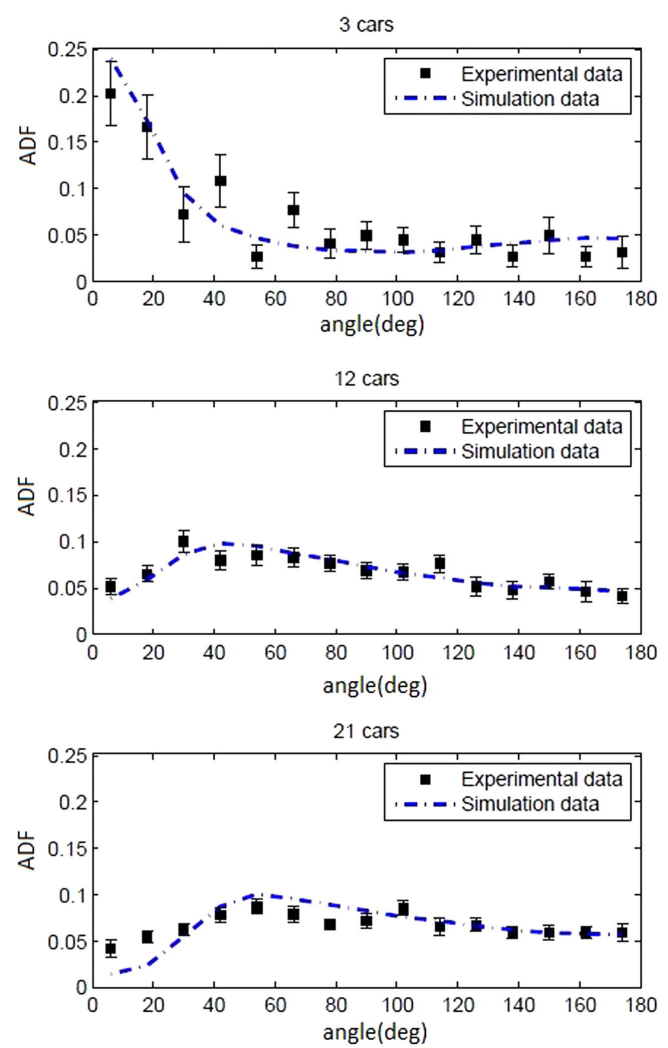

Figure 4. $\mathrm{ADF}$ for 3 (top), 12 (middle) and 21 (bottom) cars. Dash-dotted lines correspond to the simulations.

portion of the ring contained inside the track limits must be taken into account to normalize. The bins in the histogram correspond to a ring with a width of $150 \mathrm{~cm}$.

Similarly to the RDF, the angular distribution function (ADF) was obtained by computing, for each car, the angle formed by the two closest ones; the results are binned into a histogram as well. However, note that in this case no normalization is applied, since we are not counting particles but calculating angles.

Error bars are calculated using block averaging. This is done to ensure no correlation between the distribution functions at different (typically consecutive) times, over which we average. Informally, this correlation would imply that the system of particles is in a similar configuration from one evaluation to the next. This can happen (and does, in some cases, as shown by a computation of the auto-correlation) if the density is high enough to cause a very slow movement on average (i.e. collisions are frequent), so that the time step is not sufficient for the system to change significantly. If these correlations appear, the error bars that would be calculated in the standard way would be misleadingly small.

All the data analysis, as well as the simulations, have been carried out using Matlab. 


\section{Discussion}

Figure 3 shows some examples of the obtained RDFs for 3, 12 and 21 cars; similar results are obtained for an intermediate number of cars (see supplementary information, available online at stacks.iop.org/EJP/38/035802/mmedia). An immediately noticeable effect (in both the real and the simulated systems) is the decay of the RDF at long distances. As for the case of liquids, this magnitude should tend to 1 as the distance tends to infinity, since particles that are separated by a large enough distance are uncorrelated. The decay that appears in our system, instead, is a direct consequence of the finite size of our experiment: as the distance increases, there are fewer cars that contribute to the RDF since the only cars that can contribute to values significantly larger than the dimensions of the track are those near the corners. However, those cars at the corners have other cars at small distances as well, meaning that there will always be many more counts at smaller distances. This is schematically illustrated in figure 2. In short, this decay is an edge effect that stems from the fact that the system is finite. The contrary happens on most molecular dynamics simulations: periodic boundary conditions are applied making the system essentially infinite. For this reason we have decided to simulate a system as simple as possible that only captures the geometrical features of our experiment: a two-dimensional hard disks system confined in a rectangle (technical details might be found in the Methods section). Aside from this, it is important to remark that the RDFs show no significant changes to the shape of the curves in the wide range of densities studied.

As figure 3 shows, the agreement between the RDFs of the experimental data and the simulations is almost perfect within the error for all cases. A more careful look at figure 3 and at the results appearing in the supplementary material, however, reveals that in the case of low densities the density of bumper cars at short distances is above the one calculated by our simple model. This effect can be related to groups of people actively striving for collisions. However, in order to unambiguously assign this effect to conscious driving of the bumper cars, some additional experiments must be performed giving some directions to the participants driving the bumper cars. Note as well that the error bars decrease as $N$ increases, which is to be expected since the results are averaged over a larger number of data points.

We have also calculated the ADF both from the experiment and the simulation. The analysis of the results (relevant examples are shown in figure 4) reveals that, for a low number of cars, there is a clear majority of small angles. An intuitive explanation of this involves imagining only three particles (which is the minimum number to calculate an angular distribution). In this situation, a large angle (close to $180^{\circ}$ ) can only appear when the three particles are almost aligned; however, this configuration implies two counts of a near-zero angle per each count of a large one. Therefore, one can expect the observed preponderance of small angles.

On the other hand, when the number of cars becomes large enough, there is an exclusion, or minority, of small angles. The video clips show that, for such large densities, most cars are in contact with each other. This explains the absence of small angles: when particles that occupy a given space are packed together, there is a minimum possible value for the angle that can be formed between the lines joining their centres, simply because they have to remain at a minimum distance. In fact, the ADFs for the largest numbers of cars tend to show a maximum at $60^{\circ}$, consistent with hexagonal packing. Moreover, remarkably enough, as with the case for the RDF, the experimental data and the data obtained from the simulations show almost identical behaviour within the errors. 


\section{Conclusions}

Overall, the main conclusion is that simulated and experimental data produce very similar radial and angular distribution functions. The differences between them are likely to be mostly due to the lack of repetition of the experiment. However, an excess of bumper car density at low densities can be explained by groups of people striving for collisions. In any case new experiments giving different guidelines to different groups of participants are desirable to investigate if more subtle effects of human behaviour can be quantified.

The simulations have been carried out without taking into account any complex dynamic interactions, but only geometrical constraints on the system. The resemblance between the distribution functions suggests that, in complex systems like this one, the macroscopic structural properties depend mostly on general geometric restrictions, rather than on the details of local interactions. This is not trivial to deduce from the dynamics of the systems alone. In other words, the fact that cars are driven by conscious human beings does not appear to have any important effect on the structures that arise, in agreement with, and complementing, the conclusion of other works which study dynamical aspects of similar systems $[1,2,4]$ : from the structure point of view, the system behaves as if the movement was exclusively driven by random collisions.

\section{Acknowledgments}

We would like to thank the amusement park TIBIDABO for support in the performance of the experiment. We would also like to thank Artur Paz for his support on the logistics of the experiment. This work was partially supported by the Spanish Ministry of Science and Innovation through projects FIS2014-54734-P and MAT2012-33633 and by the Generalitat de Catalunya under project 2014 SGR-581.

\section{References}

[1] Moussaïd M, Helbing D, Garnier S, Johansson A, Combe M and Theraulaz G 2009 Experimental study of the behavioural mechanisms underlying self-organization in human crowds Proc. $R$. Soc. B 2762755

[2] Moussaïd M, Helbing D and Theraulaz G 2011 How simple rules determine pedestrian behaviour and crowd disasters Proc. Natl Acad. Sci. USA 1086884

[3] Farkas I, Helbing D and Vicsek T 2002 Mexican waves in an excitable medium Nature 419131

[4] Silverberg J L, Bierbaum M, Sethna J P and Cohen I 2013 Collective motion of humans in mosh and circle pits at heavy metal concerts Phys. Rev. Lett. 110228701

[5] Helbing D, Johansson A and Al-Abideen H Z 2007 Dynamics of crowd disasters: an empirical study Phys. Rev. E 75046109

[6] Helbing D, Farkas I and T Vicsek T 2000 Simulating dynamical features of escape panic Nature 407487

[7] Isobe M, Helbing D and Nagatani T 2004 Experiment, theory, and simulation of the evacuation of a room without visibility Phys. Rev. E 69066132

[8] Prusty M, Leaw J N, Chong S S and Cheong S A 2012 Stochastic boundary condtions for grandcanonical-like molecular dynamics simulations of open systems Comput. Phys. Commun. 183486

[9] Siderius D W and Gelb L D 2009 Thermodynamic and structural properties of finely discretized onlattice hard-sphere fluids: virial coefficients, free energies, and direct correlation functions J. Chem. Phys. 131084503 